\title{
Comparison of Autof ms1000 and Bruker Biotyper MALDI-TOF MS Platforms for Routine Identification of Clinical Microorganisms
}

\author{
Jae Hyeon Park $\mathbb{D}^{1,2}$ Yujin Jang, ${ }^{3}$ Inseon Yoon, ${ }^{2}$ Taek Soo Kim $\mathbb{D}^{1,},{ }^{1,2}$ \\ and Hyunwoong Park $\mathbb{D}^{1,4}$ \\ ${ }^{1}$ Department of Laboratory Medicine, Seoul National University College of Medicine, 03080 Seoul, Republic of Korea \\ ${ }^{2}$ Department of Laboratory Medicine, Seoul National University Hospital, 03080 Seoul, Republic of Korea \\ ${ }^{3}$ Biomedical Research Institute, Seoul National University Hospital, 03080 Seoul, Republic of Korea \\ ${ }^{4}$ Department of Laboratory Medicine, Seoul National University Boramae Medical Center, 07061 Seoul, Republic of Korea
}

Correspondence should be addressed to Taek Soo Kim; kim.taeksoo@snu.ac.kr

Received 5 October 2020; Revised 1 February 2021; Accepted 10 February 2021; Published 4 March 2021

Academic Editor: Stanley Brul

Copyright ( 2021 Jae Hyeon Park et al. This is an open access article distributed under the Creative Commons Attribution License, which permits unrestricted use, distribution, and reproduction in any medium, provided the original work is properly cited.

\begin{abstract}
Matrix-assisted laser desorption ionization-time of flight mass spectrometry (MALDI-TOF MS) is widely used in clinical microbiology laboratories because it is cost-effective, reliable, and fast. This study is aimed at comparing the identification performance of the recently developed Autof ms1000 (Autobio, China) with that of the Bruker Biotyper (Bruker Daltonics, Germany). From January to June 2020, 205 preserved strains and 302 clinical isolates were used for comparison. Bacteria were tested with duplicates of the direct transfer method, and formic acid extraction was performed if the results were not at the species level. Fungi were tested with formic acid extraction followed by ethanol extraction methods. 16S rRNA or ITS region sequence analysis was performed on isolates that could not be identified by any of the instruments and on isolates that showed inconsistent results. The time to result of each instrument was also compared. Among preserved strains, species-level identification results were obtained in 202 (98.5\%) strains by the Autof ms1000 and 200 (97.6\%) strains by the Bruker Biotyper. Correct identification at the species/complex level was obtained for 200 (97.6\%) strains by the Autof ms1000 and for 199 (97.1\%) strains by the Bruker Biotyper. Among clinical isolates, species-level identification results were obtained in 301 (99.7\%) strains and 300 (99.3\%) strains by the Autof ms1000 and Bruker Biotyper, respectively. Correct identification at the species/complex level was achieved for 299 (99.0\%) strains by the Autof ms1000 and for 300 (99.3\%) strains by the Bruker Biotyper. The time to analyze 96 spots was approximately $14 \mathrm{~min}$ for the Autof ms1000 and approximately 27 min for the Bruker Biotyper. The two instruments showed comparable performance for the routine identification of clinical microorganisms. In addition, the Autof ms1000 has a short test time, making it convenient for use in clinical microbiology laboratories.
\end{abstract}

\section{Introduction}

The identification of microorganisms relies on phenotypic and biochemical characteristics. The process of identification has been automated but remains laborious and time consuming. Currently, molecular identification is the standard method, but it is not routinely used in clinical microbiology laboratories due to cost and labour requirements. Matrixassisted laser desorption ionization-time of flight mass spectrometry (MALDI-TOF MS) has been rapidly introduced into laboratories because it is a rapid, economical, and accurate method for the routine identification of various microorganisms $[1,2]$. The first developed Bruker Biotyper (Bruker Daltonik GmbH, Leipzig, Germany) and VITEK MS 
(bioMérieux, Marcy l'Etoile, France) received FDA and CEmarked IVD approval. Their identification performances in clinical microbiology laboratories have been evaluated in several countries [3-8]. The strengths and limitations of MALDI-TOF MS have also been reported [1, 2]. Therefore, many laboratories are changing their routine identification methods from biochemical methods to MALDITOF MS. Since these events, various MALDI-TOF MS instruments with similar principles have been developed. These new instruments, such as the MicroIDSys system (ASTA corp., Suwon, South Korea) [9-11], Microtyper MALDI-TOF MS (Xiamen Mass Spectrometry, Xiamen, China) [12], and Clin-TOF (Bioyong Technologies, Beijing, China) [13], have been proven to be comparable to conventional MALDI-TOF MS.

The Autof ms1000 (Autobio Diagnostics in Zhengzhou, China) is a MALDI-TOF MS instrument developed in April 2018 that received CE-marked IVD clearance in June 2018. It is characterized by the use of the latest cloud database and an external pump to achieve vacuum rapidly. The use of the Autof ms1000 has been reported in a case of Trichosporon dohaense infection [14], a novel isolate of Legionella qingyii [15], and an evaluation in Bacteroides fragilis group isolates [16]. Recently, the Autof ms 1000 was evaluated for clinical isolates in China, and it showed comparable identification performance to the Bruker Biotyper [17]. Since it was tested on commonly isolated microorganisms, a comprehensive comparison was insufficient. In addition, the performance of the Autof ms 1000 for routine identification in clinical microbiology laboratories outside China has not been evaluated.

This study is aimed at comparing the diagnostic performance of the Autof ms1000 with that of the Bruker Biotyper. The identification performance was evaluated in preserved strains and clinical isolates, and the analysis time was compared.

\section{Materials and Methods}

2.1. Evaluated Microorganisms. The evaluation was divided into the use of preserved strains for the overall performance on various species and of clinical isolates for performance in routine isolates. A total of 205 strains stored in the clinical microbiology laboratory of Seoul National University Hospital from 2015 to 2020 were used as preserved strains. They included strains isolated from clinical samples and American Type Culture Collection (ATCC) strains. Clinical isolates were cultured from routine specimens received from February 2020 to June 2020. A total of 302 clinical isolates was tested, including genitourinary, respiratory, blood, gastrointestinal, and other specimens. This study was approved by the institutional review board of Seoul National University Hospital (IRB No. 1911-109-1080).

2.2. MALDI-TOF MS Analysis. The frozen aerobic bacteria and yeast strains were subcultured twice on blood agar plates (BAPs) or on Sabouraud dextrose agar. The anaerobic bacteria were subcultured twice on Brucella agar in an anaerobic jar. After the culture, in parallel, the same medical technician took the colony from the same medium and placed it on the target plate. Bacteria and fungi were transferred to the target plate by the direct transfer method and the formic acid (FA) method. Initial analysis of each strain was performed in duplicate, and the results with a higher range/score were used. If the results of both instruments were not specieslevel identification, further tests were conducted. For further tests, FA extraction was used for bacteria, and ethanol/formic acid (EtOH/FA) extraction was performed for fungi.

2.3. Autobio Autof ms1000. All procedures were performed according to the manufacturer's instructions. The test was performed using Autobio Autof ms1000, and Acquirer 1.0.151 and local DB version 1.1.11 were used to analyze the results. The database has mass spectra of 14,174 strains and 4,226 species. The Autof ms1000 instrument was calibrated daily using the calibrator. Internal quality control was performed with Staphylococcus aureus, Escherichia coli, Candida albicans, and a blank spot. The target plate of Autof ms 1000 is disposable and has 96 spots. In the direct transfer procedure, a single colony was smeared directly onto the target plate. After drying, $1 \mu \mathrm{L}$ of matrix solution ( $\alpha$-cyano- 4 hydroxycinnamic acid, CHCA) was applied to the target plate and dried at room temperature. In the FA extraction method, a single colony was smeared onto the target plate, $1 \mu \mathrm{L}$ of $70 \%$ FA was applied and dried, and then the matrix solution was applied. The EtOH/FA extraction method was performed as follows. A single colony was mixed with $300 \mu \mathrm{L}$ of HPLC grade deionized water and $900 \mu \mathrm{L}$ of ethanol in a $1.5 \mathrm{~mL}$ Eppendorf tube. After vortexing, the Eppendorf tube was centrifuged for $2 \mathrm{~min}$ at 13,000 rpm, and the supernatant was removed. The pellet was air-dried and resuspended in $10 \mu \mathrm{L}$ of FA. After vortexing, $10 \mu \mathrm{L}$ of acetonitrile $(\mathrm{ACN})$ was added, and the tube was vortexed and then centrifuged for $2 \mathrm{~min}$ at 13,000 rpm. One microlitre of the supernatant was then applied to the target plate and dried at room temperature. Then, $1 \mu \mathrm{L}$ of the matrix solution was applied to the target plate and dried at room temperature. Each result is listed in a ranking table of the identification results and range values. The identification result was interpreted according to the manufacturer's recommendations as "reliable species identification" for range 9.00010.000, "reliable genus identification" for range 6.000-8.999, and "no reliable identification" for range $<6.000$.

2.4. Bruker Biotyper. All procedures were performed according to the manufacturer's instructions. Mass spectrometry was performed using a Bruker microflex LT. The laser source has not yet been replaced. MALDI Biotyper RTC software 3.1 and 6903 MSP (main spectra) Library were used for analysis. A Bruker microflex LT instrument was calibrated weekly using the bacterial test standard (BTS, Bruker Daltonics). Internal quality control was performed with S. aureus, E. coli, C. albicans, and a blank spot. The procedures of Bruker Biotyper in the direct transfer method, FA extraction method, and EtOH/FA extraction method were similar to those of Autof ms1000. The difference was that the volume of FA and $\mathrm{ACN}$ was $50 \mu \mathrm{L}$ in EtOH/FA extraction. Each result is listed in a ranking table of the identification results and score 
TABLE 1: Identification results of preserved strains.

\begin{tabular}{|c|c|c|c|c|c|c|c|}
\hline \multirow{2}{*}{ Group of organisms } & \multirow{2}{*}{$N$} & \multicolumn{3}{|c|}{ Autof ms 1000} & \multicolumn{3}{|c|}{ Bruker Biotyper } \\
\hline & & Species (\%) & Genus (\%) & No reliable ID (\%) & Species (\%) & Genus (\%) & No reliable ID (\%) \\
\hline Enterobacterales & 59 & $59(100.0)$ & $0(0.0)$ & $0(0.0)$ & $59(100.0)$ & $0(0.0)$ & $0(0.0)$ \\
\hline Nonfermenters & 26 & $26(100.0)$ & $0(0.0)$ & $0(0.0)$ & $26(100.0)$ & $0(0.0)$ & $0(0.0)$ \\
\hline Other Gram-negative rods & 6 & $6(100.0)$ & $0(0.0)$ & $0(0.0)$ & $6(100.0)$ & $0(0.0)$ & $0(0.0)$ \\
\hline Gram-positive cocci & 77 & $77(100.0)$ & $0(0.0)$ & $0(0.0)$ & $75(97.4)$ & $2(2.6)$ & $0(0.0)$ \\
\hline Gram-positive rods & 3 & $3(100.0)$ & $0(0.0)$ & $0(0.0)$ & $3(100.0)$ & $0(0.0)$ & $0(0.0)$ \\
\hline Anaerobes & 12 & $11(91.7)$ & $1(8.3)$ & $0(0.0)$ & $12(100.0)$ & $0(0.0)$ & $0(0.0)$ \\
\hline Fungi & 22 & $20(90.9)$ & $0(0.0)$ & $2(9.1)$ & $19(86.4)$ & $3(13.6)$ & $0(0.0)$ \\
\hline Total & 205 & $202(98.5)$ & $1(0.5)$ & $2(1.0)$ & $200(97.6)$ & $5(2.4)$ & $0(0.0)$ \\
\hline
\end{tabular}

Abbreviation: ID = identification.

values. The identification result was interpreted according to the manufacturer's recommendations as "reliable species identification" for score 2.00-3.00, "reliable genus identification" for score 1.70-1.99, and "no reliable identification" for score $<1.7$.

2.5. Comparison of the Identification Results. The agreement of the results was compared at the species/complex level and the genus level. The species-level agreement was the same species-level result obtained by both MALDI-TOF MS instrument methods, and due to the limitations of MALDITOF MS, bacteria that were known to be difficult to identify accurately at the species level were compared at the complex level. Acinetobacter baumannii complex [18, 19] (A. baumannii, A. pittii, A. nosocomialis, A. lactucae, and A. calcoaceticus), Citrobacter freundii complex [20] (C. freundii, C. koseri, C. braakii, C. youngae, C. werkmanii, C. sedlakii, C. amalonaticus, and C. farmeri), Enterobacter clocae complex [21] (E. cloacae, E. asburiae, E. hormaechei, E. kobei, E. ludwigii, and E. nimipressuralis), Klebsiella pneumoniae complex [22] (K. pneumoniae, K. quasipneumoniae, and K. variicola), and Burkholderia cepacia complex [23] (B. cepacia, B. cenocepacia, B. metallica, etc.) were compared in complex results. The genus-level agreement was the same genus-level result obtained by both MALDI-TOF MS instruments.

2.6. Molecular Identification of Discrepant Results. If the two devices' results were both at the species level but did not match, sequence analyses were performed at Macrogen (Seoul, South Korea). Bacteria were subjected to $16 \mathrm{~S}$ rRNA sequencing and fungi to internal transcription spacer (ITS) region sequencing. The primers used to amplify the $16 \mathrm{~S}$ rRNA gene of the isolate were $5^{\prime}$-AGAGTTTGATCMTGGCTCAG- $3^{\prime}$ (27F) and $5^{\prime}$ -TACGGYTACCTTGTTACGACTT-3' (1492R), and the primers used for fungi were $5^{\prime}$-TCCGTAGGTGAACCTG CGG-3' (ITS1) and $5^{\prime}$-TCCTCCGCTTATTGATATGC- $3^{\prime}$ (ITS4). The sequences were compared with sequences in the GenBank database using BLAST software (https://blast.ncbi .nlm.nih.gov/Blast.cgi) and interpreted according to CLSI guidelines [24].

2.7. Comparison of the Time to Analyze. To compare the devices equally, a worklist was prepared in advance, and the time taken for the actual analysis was measured. After mounting the target plate to the device, the time to load a target plate and make a vacuum to prepare for starting the test and the time to analyze spots and report results were measured. The number of tested spots was divided into 12, reflecting actual use in laboratories and 96 in total.

2.8. Statistical Analysis. Descriptive variables are presented as frequencies, and continuous variables are presented as medians and interquartile ranges (IQRs). Groups were compared using the McNemar test for categorical characteristics and the Mann-Whitney test for continuous characteristics. $P<0.05$ was considered statistically significant. Statistical analyses were conducted using $\mathrm{R}$ software version 4.0.0 ( $\mathrm{R}$ Project for Statistical Computing, Vienna, Austria).

\section{Results}

3.1. Identification Results in Preserved Strains. Identification performance was evaluated in 205 preserved strains, including 193 clinical strains and 12 ATCC strains. They included 91 Gram-negatives, 80 Gram-positives, 12 anaerobes, and 22 fungi. The strains comprised 29 (14.1\%) Staphylococcus, 23 (11.2\%) Streptococcus, 22 (10.7\%) Candida, 21 (10.2\%) Enterococcus, 12 (5.8\%) Klebsiella, 11 (5.3\%) Enterobacter, 9 (4.4\%) Escherichia, etc.

The MALDI-TOF MS results of the Autof ms1000 and Bruker Biotyper are summarized in Table 1. The Autof ms1000 achieved species-level identification for 202 (98.5\%) strains, genus-level identification for $1(0.5 \%)$ strain, and no reliable results for $2(1.0 \%)$ strains. The Bruker Biotyper achieved species-level identification for 200 (97.6\%) strains and genus-level identification for $5(2.4 \%)$ strains. For the Autof ms1000, the 3 nonspecies results were $1(8.3 \%$, genus identification) strain of anaerobes and $2(9.1 \%$, no reliable identification) strains of fungi. For the Bruker Biotyper, the 5 nonspecies results were $2(2.6 \%$, genus identification) strains in Gram-positive cocci and 3 (13.6\%, genus identification) strains in fungi. The data of preserved strains is presented in File S1 of the Supplementary Material (available here).

3.2. Comparison of Identification Performance in Preserved Strains. Comparing the overall identification performance, the Autof ms 1000 correctly identified 200 strains at the 
TABLE 2: Comparison of identification performance in preserved strains.

\begin{tabular}{|c|c|c|c|c|c|c|c|c|c|}
\hline \multirow{2}{*}{ Species } & \multirow{2}{*}{$N$} & \multicolumn{4}{|c|}{ Autof ms 1000} & \multicolumn{4}{|c|}{ Bruker Biotyper } \\
\hline & & ID species & ID genus & No ID & MisID species & ID species & ID genus & No ID & MisID species \\
\hline Enterobacterales (\%) & 59 & $58(98.3)$ & $0(0.0)$ & $0(0.0)$ & $1(1.7)$ & $58(98.3)$ & $0(0.0)$ & $0(0.0)$ & $1(1.7)$ \\
\hline Citrobacter freundii complex & 6 & 6 & 0 & 0 & 0 & 6 & 0 & 0 & 0 \\
\hline Enterobacter aerogenes & 3 & 3 & 0 & 0 & 0 & 3 & 0 & 0 & 0 \\
\hline Enterobacter cloacae complex & 8 & 8 & 0 & 0 & 0 & 8 & 0 & 0 & 0 \\
\hline Escherichia coli & 9 & 9 & 0 & 0 & 0 & 9 & 0 & 0 & 0 \\
\hline Hafnia alvei & 1 & 1 & 0 & 0 & 0 & 1 & 0 & 0 & 0 \\
\hline Klebsiella oxytoca & 3 & 3 & 0 & 0 & 0 & 3 & 0 & 0 & 0 \\
\hline Klebsiella pneumoniae complex & 9 & 9 & 0 & 0 & 0 & 9 & 0 & 0 & 0 \\
\hline Morganella morganii & 3 & 3 & 0 & 0 & 0 & 3 & 0 & 0 & 0 \\
\hline Pantoea agglomerans & 1 & 1 & 0 & 0 & 0 & 1 & 0 & 0 & 0 \\
\hline Proteus mirabilis & 5 & 5 & 0 & 0 & 0 & 5 & 0 & 0 & 0 \\
\hline Proteus vulgaris & 2 & 2 & 0 & 0 & 0 & 2 & 0 & 0 & 0 \\
\hline Providencia rettgeri & 1 & 1 & 0 & 0 & 0 & 1 & 0 & 0 & 0 \\
\hline Providencia stuartii & 1 & 1 & 0 & 0 & 0 & 1 & 0 & 0 & 0 \\
\hline Salmonella species & 2 & 2 & 0 & 0 & 0 & 2 & 0 & 0 & 0 \\
\hline Serratia liquefaciens & 1 & 1 & 0 & 0 & 0 & 1 & 0 & 0 & 0 \\
\hline Serratia marcescens & 2 & 2 & 0 & 0 & 0 & 2 & 0 & 0 & 0 \\
\hline Serratia nematodiphila & 1 & 0 & 0 & 0 & 1 & 0 & 0 & 0 & 1 \\
\hline Yersinia enterocolitica & 1 & 1 & 0 & 0 & 0 & 1 & 0 & 0 & 0 \\
\hline Nonfermenters (\%) & 26 & $25(96.2)$ & $0(0.0)$ & $0(0.0)$ & $1(3.8)$ & $26(100.0)$ & $0(0.0)$ & $0(0.0)$ & $0(0.0)$ \\
\hline Achromobacter xylosoxidans & 2 & 2 & 0 & 0 & 0 & 2 & 0 & 0 & 0 \\
\hline Acinetobacter baumannii complex & 6 & 6 & 0 & 0 & 0 & 6 & 0 & 0 & 0 \\
\hline Acinetobacter bereziniae & 1 & 0 & 0 & 0 & 1 & 1 & 0 & 0 & 0 \\
\hline Burkholderia cepacia complex & 2 & 2 & 0 & 0 & 0 & 2 & 0 & 0 & 0 \\
\hline Moraxella catarrhalis & 2 & 2 & 0 & 0 & 0 & 2 & 0 & 0 & 0 \\
\hline Pseudomonas aeruginosa & 8 & 8 & 0 & 0 & 0 & 8 & 0 & 0 & 0 \\
\hline Pseudomonas stutzeri & 1 & 1 & 0 & 0 & 0 & 1 & 0 & 0 & 0 \\
\hline Stenotrophomonas maltophilia & 4 & 4 & 0 & 0 & 0 & 4 & 0 & 0 & 0 \\
\hline Other Gram-negative rods (\%) & 6 & $6(100.0)$ & $0(0.0)$ & $0(0.0)$ & $0(0.0)$ & $6(100.0)$ & $0(0.0)$ & $0(0.0)$ & $0(0.0)$ \\
\hline Campylobacter jejuni & 1 & 1 & 0 & 0 & 0 & 1 & 0 & 0 & 0 \\
\hline Haemophilus influenzae & 3 & 3 & 0 & 0 & 0 & 3 & 0 & 0 & 0 \\
\hline Haemophilus parainfluenzae & 1 & 1 & 0 & 0 & 0 & 1 & 0 & 0 & 0 \\
\hline Neisseria gonorrhoeae & 1 & 1 & 0 & 0 & 0 & 1 & 0 & 0 & 0 \\
\hline Gram-positive cocci (\%) & 77 & $77(100.0)$ & $0(0.0)$ & $0(0.0)$ & $0(0.0)$ & $75(97.4)$ & $2(2.6)$ & $0(0.0)$ & $0(0.0)$ \\
\hline Aerococcus viridans & 1 & 1 & 0 & 0 & 0 & 1 & 0 & 0 & 0 \\
\hline Enterococcus avium & 1 & 1 & 0 & 0 & 0 & 1 & 0 & 0 & 0 \\
\hline Enterococcus casseliflavus & 2 & 2 & 0 & 0 & 0 & 2 & 0 & 0 & 0 \\
\hline Enterococcus faecalis & 8 & 8 & 0 & 0 & 0 & 8 & 0 & 0 & 0 \\
\hline Enterococcus faecium & 9 & 9 & 0 & 0 & 0 & 9 & 0 & 0 & 0 \\
\hline Enterococcus raffinosus & 1 & 1 & 0 & 0 & 0 & 1 & 0 & 0 & 0 \\
\hline Micrococcus luteus & 1 & 1 & 0 & 0 & 0 & 1 & 0 & 0 & 0 \\
\hline Parvimonas micra & 1 & 1 & 0 & 0 & 0 & 1 & 0 & 0 & 0 \\
\hline Rothia mucilaginosa & 1 & 1 & 0 & 0 & 0 & 1 & 0 & 0 & 0 \\
\hline Staphylococcus aureus & 10 & 10 & 0 & 0 & 0 & 10 & 0 & 0 & 0 \\
\hline Staphylococcus capitis & 2 & 2 & 0 & 0 & 0 & 2 & 0 & 0 & 0 \\
\hline Staphylococcus epidermidis & 9 & 9 & 0 & 0 & 0 & 9 & 0 & 0 & 0 \\
\hline Staphylococcus haemolyticus & 3 & 3 & 0 & 0 & 0 & 3 & 0 & 0 & 0 \\
\hline Staphylococcus hominis & 1 & 1 & 0 & 0 & 0 & 1 & 0 & 0 & 0 \\
\hline
\end{tabular}


TABLE 2: Continued.

\begin{tabular}{|c|c|c|c|c|c|c|c|c|c|}
\hline \multirow{2}{*}{ Species } & \multirow{2}{*}{$N$} & \multicolumn{4}{|c|}{ Autof ms 1000} & \multicolumn{4}{|c|}{ Bruker Biotyper } \\
\hline & & ID species & ID genus & No ID & MisID species & ID species & ID genus & No ID & MisID species \\
\hline Staphylococcus lugdunensis & 1 & 1 & 0 & 0 & 0 & 1 & 0 & 0 & 0 \\
\hline Staphylococcus pasteuri & 1 & 1 & 0 & 0 & 0 & 1 & 0 & 0 & 0 \\
\hline Staphylococcus saprophyticus & 2 & 2 & 0 & 0 & 0 & 2 & 0 & 0 & 0 \\
\hline Streptococcus agalactiae & 6 & 6 & 0 & 0 & 0 & 6 & 0 & 0 & 0 \\
\hline Streptococcus anginosus & 3 & 3 & 0 & 0 & 0 & 3 & 0 & 0 & 0 \\
\hline Streptococcus constellatus & 2 & 2 & 0 & 0 & 0 & 2 & 0 & 0 & 0 \\
\hline Streptococcus dysgalactiae & 1 & 1 & 0 & 0 & 0 & 1 & 0 & 0 & 0 \\
\hline Streptococcus gallolyticus & 1 & 1 & 0 & 0 & 0 & 1 & 0 & 0 & 0 \\
\hline Streptococcus intermedius & 1 & 1 & 0 & 0 & 0 & 1 & 0 & 0 & 0 \\
\hline Streptococcus oralis & 1 & 1 & 0 & 0 & 0 & 1 & 0 & 0 & 0 \\
\hline Streptococcus parasanguinis & 1 & 1 & 0 & 0 & 0 & 0 & 1 & 0 & 0 \\
\hline Streptococcus pneumoniae & 2 & 2 & 0 & 0 & 0 & 1 & 1 & 0 & 0 \\
\hline Streptococcus pyogenes & 3 & 3 & 0 & 0 & 0 & 3 & 0 & 0 & 0 \\
\hline Streptococcus salivarius & 2 & 2 & 0 & 0 & 0 & 2 & 0 & 0 & 0 \\
\hline Gram-positive rods (\%) & 3 & $3(100.0)$ & $0(0.0)$ & $0(0.0)$ & $0(0.0)$ & $3(100.0)$ & $0(0.0)$ & $0(0.0)$ & $0(0.0)$ \\
\hline Corynebacterium striatum & 2 & 2 & 0 & 0 & 0 & 2 & 0 & 0 & 0 \\
\hline Listeria monocytogenes & 1 & 1 & 0 & 0 & 0 & 1 & 0 & 0 & 0 \\
\hline Anaerobes (\%) & 12 & $11(91.7)$ & $1(8.3)$ & $0(0.0)$ & $0(0.0)$ & $12(100.0)$ & $0(0.0)$ & $0(0.0)$ & $0(0.0)$ \\
\hline Bacteroides fragilis & 2 & 2 & 0 & 0 & 0 & 2 & 0 & 0 & 0 \\
\hline Bacteroides ovatus & 1 & 1 & 0 & 0 & 0 & 1 & 0 & 0 & 0 \\
\hline Bacteroides thetaiotaomicron & 1 & 1 & 0 & 0 & 0 & 1 & 0 & 0 & 0 \\
\hline Clostridioides difficile & 1 & 1 & 0 & 0 & 0 & 1 & 0 & 0 & 0 \\
\hline Clostridium perfringens & 1 & 1 & 0 & 0 & 0 & 1 & 0 & 0 & 0 \\
\hline Eggerthella lenta & 1 & 0 & 1 & 0 & 0 & 1 & 0 & 0 & 0 \\
\hline Fusobacterium nucleatum & 1 & 1 & 0 & 0 & 0 & 1 & 0 & 0 & 0 \\
\hline Prevotella melaninogenica & 1 & 1 & 0 & 0 & 0 & 1 & 0 & 0 & 0 \\
\hline Propionibacterium acnes & 2 & 2 & 0 & 0 & 0 & 2 & 0 & 0 & 0 \\
\hline Veillonella parvula & 1 & 1 & 0 & 0 & 0 & 1 & 0 & 0 & 0 \\
\hline Fungi (\%) & 22 & $20(90.9)$ & $0(0.0)$ & $2(9.1)$ & $0(0.0)$ & $19(86.4)$ & $3(13.6)$ & $0(0.0)$ & $0(0.0)$ \\
\hline Candida albicans & 5 & 5 & 0 & 0 & 0 & 5 & 0 & 0 & 0 \\
\hline Candida auris & 2 & 0 & 0 & 2 & 0 & 1 & 1 & 0 & 0 \\
\hline Candida dubliniensis & 1 & 1 & 0 & 0 & 0 & 1 & 0 & 0 & 0 \\
\hline Candida glabrata & 3 & 3 & 0 & 0 & 0 & 2 & 1 & 0 & 0 \\
\hline Candida krusei & 2 & 2 & 0 & 0 & 0 & 2 & 0 & 0 & 0 \\
\hline Candida orthopsilosis & 1 & 1 & 0 & 0 & 0 & 0 & 1 & 0 & 0 \\
\hline Candida parapsilosis & 3 & 3 & 0 & 0 & 0 & 3 & 0 & 0 & 0 \\
\hline Candida tropicalis & 4 & 4 & 0 & 0 & 0 & 4 & 0 & 0 & 0 \\
\hline Cryptococcus neoformans & 1 & 1 & 0 & 0 & 0 & 1 & 0 & 0 & 0 \\
\hline Total (\%) & 205 & $200(97.6)$ & $1(0.5)$ & $2(1.0)$ & $2(1.0)$ & $199(97.1)$ & $5(2.4)$ & $0(0.0)$ & $1(0.5)$ \\
\hline
\end{tabular}

Abbreviations: ID = identification; MisID = misidentification.

species/complex level and 1 strain at the genus level (Table 2). The Bruker Biotyper correctly identified 199 strains at the species/complex level and 5 strains at the genus level. There was no significant difference in the species/complex level identification performance of the two instruments $(P$ value $=1.000)$. The Autof ms1000 and Bruker Biotyper misidentified 2 strains and 1 strain, respectively. The correct results of the Autof ms1000 were Enterobacterales 98.3\% (58/59), nonfermenters $96.2 \%$ (25/26), other Gramnegative rods $100.0 \%$ (6/6), Gram-positive cocci $100.0 \%$ (77/77), Gram-positive rods 100.0\% (3/3), anaerobes $91.7 \%$ (11/12), and fungi $90.9 \%(20 / 22)$. The correct results of the Bruker Biotyper were Enterobacterales 98.3\% (58/59), nonfermenters $96.2 \%$ (25/26), other Gram-negative rods 
TABLE 3: Identification results in clinical isolates.

\begin{tabular}{lccccccc}
\hline \multirow{2}{*}{ Group of organisms } & \multirow{2}{*}{$N$} & \multicolumn{2}{c}{ Autof ms1000 } & \multicolumn{3}{c}{ Bruker Biotyper } \\
& & Species (\%) & Genus (\%) & No reliable ID (\%) & Species (\%) & Genus (\%) & No reliable ID (\%) \\
\hline Enterobacterales & 131 & $131(100.0)$ & $0(0.0)$ & $0(0.0)$ & $131(100.0)$ & $0(0.0)$ & $0(0.0)$ \\
Nonfermenters & 41 & $41(100.0)$ & $0(0.0)$ & $0(0.0)$ & $41(100.0)$ & $0(0.0)$ & $0(0.0)$ \\
Other Gram-negative rods & 2 & $2(100.0)$ & $0(0.0)$ & $0(0.0)$ & $2(100.0)$ & $0(0.0)$ & $0(0.0)$ \\
Gram-positive cocci & 118 & $118(100.0)$ & $0(0.0)$ & $0(0.0)$ & $116(98.3)$ & $2(1.7)$ & $0(0.0)$ \\
Fungi & 10 & $9(90.0)$ & $0(0.0)$ & $1(10.0)$ & $10(100.0)$ & $0(0.0)$ & $0(0.0)$ \\
Total & 302 & $301(99.7)$ & $0(0.0)$ & $1(0.3)$ & $300(99.3)$ & $2(0.7)$ & $0(0.0)$ \\
\hline
\end{tabular}

Abbreviation: ID = identification.

100.0\% (6/6), Gram-positive cocci 97.4\% (75/77), Grampositive rods $100.0 \%(3 / 3)$, anaerobes $100.0 \%(12 / 12)$, and fungi $86.4 \%(19 / 22)$.

\subsection{Identification Results in Clinical Isolates. A total of 302} clinical isolates were used for evaluation, of which 174 were Gram-negatives, 118 Gram-positives, and 10 fungi. Their genera were Staphylococcus (59, 19.5\%), Escherichia (54, 17.9\%), Enterococcus (49, 16.2\%), Klebsiella (31, 10.3\%), Enterobacter (24, 8.0\%), Pseudomonas (24, 8.0\%), Streptococcus $(10,3.3 \%)$, etc. The MALDI-TOF MS results of both instruments are presented in Table 3. The results of the Autof ms1000 were 301 (99.7\%) species-level identification and $1(0.3 \%)$ no reliable result. The results of the Bruker Biotyper were 300 (99.3\%) species-level identification and $2(0.7 \%)$ genus-level identification. No specieslevel results were obtained in $1(10.0 \%)$ yeast with no reliable results by the Autof ms1000 and 2 (1.7\%) Grampositive cocci with genus-level results by the Bruker Biotyper. The data of clinical isolates is presented in File S1 of the Supplementary Material.

3.4. Comparison of Identification Performance in Clinical Isolates. In the comparison of the overall performance, the correct results obtained by the Autof ms1000 were 299 isolates at the species/complex level, and the correct results obtained by the Bruker Biotyper were 300 isolates at the species/complex level and 2 isolates at the genus level (Table 4). There was no significant difference in the species/complexlevel identification performance of the two instruments $(P$ value $=1.000)$. The Autof ms 1000 produced 1 case with no reliable result and 2 misidentifications. There were no unreliable results or misidentifications by the Bruker Biotyper. The correct results of the Autof ms 1000 were Enterobacterales $99.2 \%$ (130/131), nonfermenters $100.0 \%$ (41/41), other Gram-negative rods $100.0 \%(2 / 2)$, Gram-positive cocci $99.2 \%$ (117/118), and fungi 90.0\% (9/10). The correct results of the Bruker Biotyper were Enterobacterales $100.0 \%$ (131/131), nonfermenters $100.0 \%$ (41/41), other Gramnegative rods $100.0 \%(2 / 2)$, Gram-positive cocci $98.4 \%$ $(116 / 118)$, and fungi $100.0 \%(10 / 10)$.

3.5. Inconsistent Results between the Autof ms1000 and the Bruker Biotyper. Discrepancies between the speciescomplex results obtained by the two instruments are summarized in Table 5 . There were 5 misidentifications produced by the Autof ms1000 and 1 by the Bruker Biotyper. The strain incorrectly identified by both instruments was $S$. nematodiphila, which was misidentified as $S$. marcescens by the Autof ms1000 and S. ureilytica by the Bruker Biotyper. E. gallinarum was misidentified as E. casseliflavus for the Autof ms1000, and the Bruker Biotyper produced the same result but at the genus level. In addition, the Autof ms1000 incorrectly identified $A$. bereziniae as A. guillouiae and K. oxytoca as $R$. ornithinolytica. Due to the low range/score values, there were 3 cases of no reliable results for the Autof ms1000, all of which were Candida auris. The Bruker Biotyper identified 2 strains of $C$. auris at the species level and 1 at the genus level. The genus-level results were 1 strain with the Autof ms 1000 and 7 strains with the Bruker Biotyper. Except for E. casseliflavus, the genus-level results were consistent with the species results.

3.6. Comparison of the Analysis Time. The target plate was placed on the device, and then, the test preparation time and the time required to analyze 12 and 96 spots were measured (Table 6). Loading the target plate and establishing vacuum took $36 \mathrm{sec}$ for the Autof ms1000 and $1 \mathrm{~min} 33 \mathrm{sec}$ for the Bruker Biotyper $(P$ value $<0.001)$. When twelve spots were analyzed, the test times for the Autof ms1000 and Bruker Biotyper were $1 \mathrm{~min} 40 \mathrm{sec}$ and $3 \mathrm{~min} 13 \mathrm{sec}$, respectively $(P$ value $<0.001)$. In addition, the Autof ms 1000 took 13 min $52 \mathrm{sec}$ to analyze 96 spots, and the Bruker Biotyper took $25 \mathrm{~min} 38 \mathrm{sec}$.

\section{Discussion}

This study showed that the species-level identification performance of the Autof ms 1000 and Bruker Biotyper in clinically significant strains is comparable at $97 \%$ or more. Considering microorganisms that are difficult to identify with MALDI-TOF MS, excellent agreement was confirmed. These results used conventional extraction methods and may be applicable to practical use in clinical microbiology laboratories.

Results were obtained at the genus level for 7 isolates by the Bruker Biotyper but for 1 isolate by the Autof ms1000. Five of these results were for Gram-positive cocci and 2 for fungi. In a comparative evaluation with the VITEK MS, the Bruker Biotyper achieved lower species-level identification rates in Gram-positive and yeast strains than the VITEK MS [6, 8]. However, when genus-level results were 
TABLE 4: Comparison of identification performance in clinical isolates.

\begin{tabular}{|c|c|c|c|c|c|c|c|c|c|}
\hline \multirow{2}{*}{ Species } & \multirow{2}{*}{$N$} & \multicolumn{4}{|c|}{ Autof ms 1000} & \multicolumn{4}{|c|}{ Bruker Biotyper } \\
\hline & & ID species & ID genus & No ID & MisID species & ID species & ID genus & No ID & MisID species \\
\hline Enterobacterales (\%) & 131 & $130(99.2)$ & $0(0.0)$ & $0(0.0)$ & $1(0.8)$ & $131(100.0)$ & $0(0.0)$ & $0(0.0)$ & $0(0.0)$ \\
\hline Citrobacter freundii complex & 6 & 6 & 0 & 0 & 0 & 6 & 0 & 0 & 0 \\
\hline Enterobacter aerogenes & 10 & 10 & 0 & 0 & 0 & 10 & 0 & 0 & 0 \\
\hline Enterobacter cloacae complex & 14 & 14 & 0 & 0 & 0 & 14 & 0 & 0 & 0 \\
\hline Escherichia coli & 54 & 54 & 0 & 0 & 0 & 54 & 0 & 0 & 0 \\
\hline Klebsiella oxytoca & 2 & 1 & 0 & 0 & 1 & 2 & 0 & 0 & 0 \\
\hline Klebsiella pneumoniae complex & 29 & 29 & 0 & 0 & 0 & 29 & 0 & 0 & 0 \\
\hline Morganella morganii & 3 & 3 & 0 & 0 & 0 & 3 & 0 & 0 & 0 \\
\hline Proteus mirabilis & 6 & 6 & 0 & 0 & 0 & 6 & 0 & 0 & 0 \\
\hline Raoultella ornithinolytica & 4 & 4 & 0 & 0 & 0 & 4 & 0 & 0 & 0 \\
\hline Serratia marcescens & 3 & 3 & 0 & 0 & 0 & 3 & 0 & 0 & 0 \\
\hline Nonfermenters (\%) & 41 & $41(100.0)$ & $0(0.0)$ & $0(0.0)$ & $0(0.0)$ & $41(100.0)$ & $0(0.0)$ & $0(0.0)$ & $0(0.0)$ \\
\hline Acinetobacter baumannii complex & 9 & 9 & 0 & 0 & 0 & 9 & 0 & 0 & 0 \\
\hline Elizabethkingia meningoseptica & 1 & 1 & 0 & 0 & 0 & 1 & 0 & 0 & 0 \\
\hline Pseudomonas aeruginosa & 24 & 24 & 0 & 0 & 0 & 24 & 0 & 0 & 0 \\
\hline Stenotrophomonas maltophilia & 7 & 7 & 0 & 0 & 0 & 7 & 0 & 0 & 0 \\
\hline Other Gram-negative rods (\%) & 2 & $2(100.0)$ & $0(0.0)$ & $0(0.0)$ & $0(0.0)$ & $2(100.0)$ & $0(0.0)$ & $0(0.0)$ & $0(0.0)$ \\
\hline Haemophilus influenzae & 2 & 2 & 0 & 0 & 0 & 2 & 0 & 0 & 0 \\
\hline Gram-positive cocci (\%) & 118 & $117(99.2)$ & $0(0.0)$ & $0(0.0)$ & $1(0.8)$ & $116(98.3)$ & $2(1.7)$ & $0(0.0)$ & $0(0.0)$ \\
\hline Enterococcus avium & 1 & 1 & 0 & 0 & 0 & 1 & 0 & 0 & 0 \\
\hline Enterococcus faecalis & 15 & 15 & 0 & 0 & 0 & 15 & 0 & 0 & 0 \\
\hline Enterococcus faecium & 32 & 32 & 0 & 0 & 0 & 32 & 0 & 0 & 0 \\
\hline Enterococcus gallinarum & 1 & 0 & 0 & 0 & 1 & 0 & 1 & 0 & 0 \\
\hline Staphylococcus aureus & 29 & 29 & 0 & 0 & 0 & 29 & 0 & 0 & 0 \\
\hline Staphylococcus capitis & 3 & 3 & 0 & 0 & 0 & 3 & 0 & 0 & 0 \\
\hline Staphylococcus caprae & 1 & 1 & 0 & 0 & 0 & 1 & 0 & 0 & 0 \\
\hline Staphylococcus epidermidis & 16 & 16 & 0 & 0 & 0 & 15 & 1 & 0 & 0 \\
\hline Staphylococcus haemolyticus & 2 & 2 & 0 & 0 & 0 & 2 & 0 & 0 & 0 \\
\hline Staphylococcus hominis & 5 & 5 & 0 & 0 & 0 & 5 & 0 & 0 & 0 \\
\hline Staphylococcus lugdunensis & 3 & 3 & 0 & 0 & 0 & 3 & 0 & 0 & 0 \\
\hline Streptococcus agalactiae & 8 & 8 & 0 & 0 & 0 & 8 & 0 & 0 & 0 \\
\hline Streptococcus anginosus & 1 & 1 & 0 & 0 & 0 & 1 & 0 & 0 & 0 \\
\hline Streptococcus pyogenes & 1 & 1 & 0 & 0 & 0 & 1 & 0 & 0 & 0 \\
\hline Fungi (\%) & 10 & $9(90.0)$ & $0(0.0)$ & $1(10.0)$ & $0(0.0)$ & $10(100.0)$ & $0(0.0)$ & $0(0.0)$ & $0(0.0)$ \\
\hline Candida albicans & 4 & 4 & 0 & 0 & 0 & 4 & 0 & 0 & 0 \\
\hline Candida auris & 1 & 0 & 0 & 1 & 0 & 1 & 0 & 0 & 0 \\
\hline Candida krusei & 3 & 3 & 0 & 0 & 0 & 3 & 0 & 0 & 0 \\
\hline Candida lusitaniae & 1 & 1 & 0 & 0 & 0 & 1 & 0 & 0 & 0 \\
\hline Rhodotorula mucilaginosa & 1 & 1 & 0 & 0 & 0 & 1 & 0 & 0 & 0 \\
\hline Total (\%) & 302 & $299(99.0)$ & $0(0.0)$ & $1(0.3)$ & $2(0.7)$ & $300(99.3)$ & $2(0.7)$ & $0(0.0)$ & $0(0.0)$ \\
\hline
\end{tabular}

Abbreviations: ID = identification; MisID = misidentification; NA = not applicable.

interpreted as species-level identification, the results were mostly consistent, and similar results were obtained $(85.7 \%$, $6 / 7$ isolates).

The Autof ms1000 provided species-level results that were actual misidentifications in 4 isolates, whereas the Bruker Biotyper provided 1. S. marcecens and S. ureilytica are known to be difficult to distinguish by MALDI-TOF MS, and S. nematodiphila is a new strain reported in 2009 and seems to be misidentified in both instruments because it is phylogenetically close to other strains [25]. The Autof ms1000 incorrectly identified A. bereziniae as A. guillouiae. Both bacteria were proposed together as novel species in 2010 and known to be phylogenetically close but can be distinguished by specific signals in MALDI-TOF MS [26]. The 
TABLE 5: Detailed results of microorganisms that either were misidentified or could not be identified by one or both MALDI-TOF MS systems.

\begin{tabular}{|c|c|c|c|c|c|c|c|}
\hline \multirow{2}{*}{ Test groups } & \multirow{2}{*}{ Reference } & \multicolumn{3}{|c|}{ Autof ms 1000} & \multicolumn{3}{|c|}{ Bruker Biotyper } \\
\hline & & Result & Range & Agreement & Result & Score & Agreement \\
\hline \multirow{9}{*}{ Preserved strains } & Streptococcus pneumoniae & S. pneumoniae & 9.400 & ID species & S. pneumoniae & 1.924 & ID genus \\
\hline & Serratia nematodiphila & S. marcescens & 9.431 & MisID species & S. ureilytica & 2.136 & MisID species \\
\hline & Candida orthopsilosis & C. orthopsilosis & 9.507 & ID species & C. orthopsilosis & 1.82 & ID genus \\
\hline & Streptococcus parasanguinis & S. parasanguinis & 9.361 & ID species & S. parasanguinis & 1.907 & ID genus \\
\hline & Candida glabrata & C. glabrata & 9.441 & ID species & C. glabrata & 1.866 & ID genus \\
\hline & Eggerthella lenta & E. lenta & 8.967 & ID genus & E. lenta & 2.114 & ID species \\
\hline & Acinetobacter bereziniae & A. guillouiae & 9.127 & MisID species & A. bereziniae & 2.177 & ID species \\
\hline & Candida auris & No reliable ID & 5.401 & No ID & C. auris & 2.031 & ID species \\
\hline & Candida auris & No reliable ID & 4.755 & No ID & C. auris & 1.892 & ID genus \\
\hline \multirow{4}{*}{ Clinical isolates } & Candida auris & No reliable ID & 4.952 & No ID & C. auris & 2.073 & ID species \\
\hline & Enterococcus gallinarum & E. casseliflavus & 9.543 & MisID species & E. casseliflavus & 1.905 & ID genus \\
\hline & Klebsiella oxytoca & Raoultella ornithinolytica & 9.159 & MisID species & K. oxytoca & 2.173 & ID species \\
\hline & Staphylococcus epidermidis & S. epidermidis & 9.508 & ID species & S. epidermidis & 1.982 & ID genus \\
\hline
\end{tabular}

Abbreviations: ID = identification; MisID = misidentification .

TABle 6: Comparison of time to identification by Autof ms1000 and Bruker Biotyper.

\begin{tabular}{|c|c|c|c|}
\hline Description & Autof ms 1000 & Bruker Biotyper & $\begin{array}{c}P \\
\text { value }\end{array}$ \\
\hline $\begin{array}{l}\text { Time to load target plate and make vacuum } \\
(n=14)\end{array}$ & $36 \mathrm{sec}(35 \mathrm{sec}-37 \mathrm{sec})$ & $1 \min 33 \mathrm{sec}(1 \min 26 \mathrm{sec}-1 \min 53 \mathrm{sec})$ & $<0.001$ \\
\hline Time to analyze 12 spots $(n=37)$ & $1 \mathrm{~min} 40 \mathrm{sec}(1 \mathrm{~min} 31 \mathrm{sec}-1 \mathrm{~min} 52 \mathrm{sec})$ & $3 \min 13 \mathrm{sec}(2 \min 58 \mathrm{sec}-3 \min 45 \mathrm{sec})$ & $<0.001$ \\
\hline Time to analyze 96 spots $(n=3)$ & $\begin{array}{c}13 \mathrm{~min} 52 \mathrm{sec}(13 \mathrm{~min} 35 \mathrm{sec}-13 \mathrm{~min} \\
55 \mathrm{sec})\end{array}$ & $\begin{array}{c}25 \mathrm{~min} 38 \mathrm{sec}(22 \mathrm{~min} 17 \mathrm{sec}-27 \mathrm{~min} \\
56 \mathrm{sec})\end{array}$ & NA \\
\hline
\end{tabular}

Abbreviation: $\mathrm{NA}=$ not applicable.

misidentification may have occurred because A. bereziniae is not included in the Autof ms 1000 database. Both devices identified E. gallinarum as E. casseliflavus, and the results were species level in the Autof ms1000 and genus level in the Bruker Biotyper. It has been reported that MALDI-TOF MS can accurately identify Enterococcus at the species level [27]. The E. gallinarum strain was considered a challenging strain to identify by MALDI-TOF MS, since both devices showed correct results for two E. casseliflavus strains. K. oxytoca was identified as R. ornithinolytica in the Autof ms1000, and these species are also known to be difficult to distinguish by MALDI-TOF MS [28].

Autof ms1000 failed to identify all three strains of $C$. auris. C. auris is an emerging pathogen in infection prevention due to reported nosocomial outbreaks and high rates of antifungal resistance [29]. In 2019, the US Centers for Disease Control and Prevention (CDC) recommended the use of MALDI-TOF MS for the identification of $C$. auris and announced available instruments and database version information. It was confirmed that $C$. auris was not included in the installed database of Autof ms1000 used in this evaluation. The manufacturer recently updated the cloud database with $C$. auris, and the spectra obtained by EtOH/FA extrac- tion were analyzed with the cloud database and correctly identified (range: 9.271-9.521).

Among the misidentifications, it was the most clinically important that the Autof ms 1000 failed to identify C. auris. As a result, appropriate measures such as infection control and antifungal prescription may be delayed [29]. Additionally, the Autof ms1000 incorrectly identified K. oxytoca as $R$. ornithinolyticus, a rare pathogen in human infection [30]. With the development of identification technology, recent reports on the pathogenicity of $R$. ornithinolyticus have been made [30]. Other species-level misidentifications, including Serratia spp., Acinetobacter spp., and Enterococcus spp., had little clinical significance, as they did not alter the interpretation of antimicrobial susceptibility [31]. An important misidentification confirmed in the recent Autof ms 1000 evaluation in China is that the Bruker Biotyper failed to identify B. pseudomallei because it was not included in the database [17].

The Autof ms1000 has the advantage of using the latest library free of charge through the cloud database. The Bruker Biotyper does not support cloud databases, and the cost of updating the database is a burden for the laboratory. In this evaluation, we used an installed database created in June 
2019 because the cloud database was unavailable due to the hospital's security policy. The real analysis of the Autof ms1000 using the latest database is performed in the cloud, and the database is not downloadable. However, as medical institutions' network security is becoming increasingly important [32], Internet access may not be possible. Therefore, it is necessary to update the MALDI-TOF MS database regularly. Unlike the Bruker Biotyper, the Autof ms1000 has the disadvantage that users cannot check the species included in the database with software. Since the role of MALDI-TOF MS has been emphasized in recent cutaneous diphtheria cases [33], it should be possible to check the database when there is a related issue.

The Autof ms1000 and Bruker Biotyper have similar ease of use. Both MALDI-TOF MS systems are benchtops, but the Autof ms1000 has an external vacuum pump, which makes more noise. Neither device provides a ready-to-use matrix solution, and the matrix solution and the calibrator are provided lyophilized. In addition, 70\% FA and ACN are used in the extraction steps for both instruments. The Autof ms 1000 provides only disposable target plates, whereas the Bruker Biotyper provides both reusable and disposable target plates.

When comparing the time for identification, the Autof ms1000 was faster than the Bruker Biotyper in both the time for loading the target plate and achieving vacuum and the time for analyzing the plate. These times were reported as 2-3 min and 40-50 min (96 spots) for the Bruker Biotyper and as 5-6 min and $45-55 \mathrm{~min}$ (96 spots) for the VITEK MS $[3,8]$. The time taken for the examination did not differ from that in the reports. The difference in time for loading the target plate and achieving vacuum seems to be due to an external pump of the Autof ms1000. Additionally, the Autof ms1000 optimized the order of reading spots and reduced moving time. For example, the Bruker Biotyper tests the A12 spot and then the B1 spot, while the Autof ms1000 tests the A12 spot and then the B12 spot. Considering using 10-20 spots at a time with MALDI-TOF MS in a clinical microbiology laboratory, the analysis time of the Autof ms 1000 is more than twice as fast as that of the Bruker Biotyper, making it convenient for laboratories. However, the impact of rapid MALDI-TOF MS on actual reporting time and workflow in laboratories should be investigated in further studies.

\section{Conclusions}

The Autof ms1000 showed comparable identification performance to that of the Bruker Biotyper. The Autof ms 1000 provided more species-level results in Gram-positive cocci and fungi than the Bruker Biotyper but produced more misidentifications due to a database problem. In particular, the test time of the Autof ms1000 was approximately half that of the Bruker Biotyper, which is helpful in providing higher throughput in the clinical microbiology laboratory.

\section{Data Availability}

The data used to support the findings of this study are included within the supplementary information file.

\section{Disclosure}

The funder had no role in study design, data collection and analysis, decision to publish, or preparation of the manuscript.

\section{Conflicts of Interest}

The authors declare that there is no conflict of interest regarding the publication of this paper.

\section{Acknowledgments}

We thank Sunghee Yoon, Bosung Scientific Co., Ltd. (Seoul, South Korea), for her assistance during this study. This study was supported by Bosung Scientific Co., Ltd. (Seoul, South Korea).

\section{Supplementary Materials}

Supplementary File 1: the MALDI-TOF MS results in preserved strains and clinical isolates. (Supplementary Materials)

\section{References}

[1] A. E. Clark, E. J. Kaleta, A. Arora, and D. M. Wolk, "Matrixassisted laser desorption ionization-time of flight mass spectrometry: a fundamental shift in the routine practice of clinical microbiology," Clinical Microbiology Reviews, vol. 26, no. 3, pp. 547-603, 2013.

[2] R. Patel, "MALDI-TOF MS for the diagnosis of infectious diseases," Clinical Chemistry, vol. 61, no. 1, pp. 100-111, 2015.

[3] D. Martiny, L. Busson, I. Wybo, R. A. El Haj, A. Dediste, and O. Vandenberg, "Comparison of the Microflex LT and Vitek MS systems for routine identification of bacteria by matrixassisted laser desorption ionization-time of flight mass spectrometry," Journal of Clinical Microbiology, vol. 50, no. 4, pp. 1313-1325, 2012.

[4] W. Jamal, M. J. Albert, and V. O. Rotimi, "Real-time comparative evaluation of bioMerieux VITEK MS versus Bruker Microflex MS, two matrix-assisted laser desorptionionization time-of-flight mass spectrometry systems, for identification of clinically significant bacteria," BMC Microbiology, vol. 14, no. 1, p. 289, 2014.

[5] K. Bilecen, G. Yaman, U. Ciftci, and Y. R. Laleli, "Performances and reliability of Bruker Microflex LT and VITEK MS MALDI-TOF mass spectrometry systems for the identification of clinical microorganisms," BioMed Research International, vol. 2015, Article ID 516410, 18 pages, 2015.

[6] E. Deak, C. L. Charlton, A. M. Bobenchik et al., "Comparison of the Vitek MS and Bruker Microflex LT MALDI-TOF MS platforms for routine identification of commonly isolated bacteria and yeast in the clinical microbiology laboratory," Diagnostic Microbiology and Infectious Disease, vol. 81, no. 1, pp. 27-33, 2015.

[7] S. Levesque, P. J. Dufresne, H. Soualhine et al., "A side by side comparison of Bruker Biotyper and VITEK MS: utility of MALDI-TOF MS technology for microorganism identification in a public health reference laboratory," PLoS One, vol. 10, no. 12, article e0144878, 2015.

[8] L. Porte, P. Garcia, S. Braun et al., "Head-to-head comparison of Microflex LT and Vitek MS systems for routine 
identification of microorganisms by MALDI-TOF mass spectrometry in Chile," PLoS One, vol. 12, no. 5, article e0177929, 2017.

[9] Y. Lee, J. Y. Sung, H. Kim, D. Yong, and K. Lee, "Comparison of a new matrix-assisted laser desorption/ionization time-offlight mass spectrometry platform, ASTA MicroIDSys, with Bruker Biotyper for species identification," Annals of Laboratory Medicine, vol. 37, no. 6, pp. 531-535, 2017.

[10] H. Lee, J. H. Park, J. Oh et al., "Evaluation of a new matrixassisted laser desorption/ionization time-of-flight mass spectrometry system for the identification of yeast isolation," Journal of Clinical Laboratory Analysis, vol. 33, no. 2, article e22685, 2019.

[11] D. Kim, S. Ji, J. R. Kim et al., "Performance evaluation of a new matrix-assisted laser desorption/ionization time-of-flight mass spectrometry, ASTA MicroIDSys system, in bacterial identification against clinical isolates of anaerobic bacteria," Anaerobe, vol. 61, article 102131, 2020.

[12] Y. Huang, M. Zhang, M. Zhu et al., "Comparison of two matrix-assisted laser desorption ionization-time of flight mass spectrometry systems for the identification of clinical filamentous fungi," World Journal of Microbiology and Biotechnology, vol. 33, no. 7, p. 142, 2017.

[13] Y. L. Huang, Q. L. Sun, J. P. Li, Y. Y. Hu, H. W. Zhou, and R. Zhang, "Evaluation of an in-house MALDI-TOF MS rapid diagnostic method for direct identification of microorganisms from blood cultures," Journal of Medical Microbiology, vol. 68, no. 1, pp. 41-47, 2019.

[14] S. Y. Yu, L. N. Guo, M. Xiao et al., "Trichosporon dohaense, a rare pathogen of human invasive infections, and literature review," Infection and Drug Resistance, vol. 11, pp. 15371547, 2018.

[15] H. Y. Wu, H. Yan, M. L. Zheng et al., "Legionella qingyii sp. nov., isolated from water samples in China," International Journal of Systematic and Evolutionary Microbiology, vol. 69, no. 7, pp. 2017-2022, 2019.

[16] Y. Wang, X. F. Chen, X. L. Xie et al., "Evaluation of VITEK MS, Clin-ToF-II MS, Autof MS 1000 and VITEK 2 ANC card for identification of Bacteroides fragilis group isolates and antimicrobial susceptibilities of these isolates in a Chinese university hospital," Journal of Microbiology, Immunology and Infection, vol. 52, no. 3, pp. 456-464, 2019.

[17] Q. Ma, Q. Zhang, Y. Yuan et al., "Evaluation of the Autof MS1000 mass spectrometer in the identification of clinical isolates," BMC Microbiology, vol. 20, no. 1, p. 318, 2020.

[18] P. R. Hsueh, L. C. Kuo, T. C. Chang et al., "Evaluation of the Bruker Biotyper matrix-assisted laser desorption ionizationtime of flight mass spectrometry system for identification of blood isolates of Acinetobacter species," Journal of Clinical Microbiology, vol. 52, no. 8, pp. 3095-3100, 2014.

[19] K. Kishii, K. Kikuchi, N. Matsuda et al., "Evaluation of matrixassisted laser desorption ionization-time of flight mass spectrometry for species identification of Acinetobacter strains isolated from blood cultures," Clinical Microbiology and Infection, vol. 20, no. 5, pp. 424-430, 2014.

[20] R. Kolinska, P. Spanelova, M. Drevinek, J. Hrabak, and H. Zemlickova, "Species identification of strains belonging to genus Citrobacter using the biochemical method and MALDI-TOF mass spectrometry," Folia Microbiologica, vol. 60, no. 1, pp. 53-59, 2015.

[21] M. Pavlovic, R. Konrad, A. N. Iwobi, A. Sing, U. Busch, and I. Huber, "A dual approach employing MALDI-TOF MS and real-time PCR for fast species identification within the Enterobacter cloacae complex," FEMS Microbiology Letters, vol. 328, no. 1, pp. 46-53, 2012.

[22] C. Rodrigues, V. Passet, A. Rakotondrasoa, and S. Brisse, "Identification of Klebsiella pneumoniae, Klebsiella quasipneumoniae, Klebsiella variicola and related phylogroups by MALDI-TOF mass spectrometry," Frontiers in Microbiology, vol. 9, p. 3000, 2018.

[23] L. C. Fehlberg, L. H. Andrade, D. M. Assis, R. H. Pereira, A. C. Gales, and E. A. Marques, "Performance of MALDI-ToF MS for species identification of Burkholderia cepacia complex clinical isolates," Diagnostic Microbiology and Infectious Disease, vol. 77, no. 2, pp. 126-128, 2013.

[24] CLSI, Interpretive Criteria for Identification of Bacteria and Fungi by Targeted DNA Sequencing, P. A. Wayne, Ed., Clinical and Laboratory Standards Institute, 2nd edition, 2018.

[25] C. X. Zhang, S. Y. Yang, M. X. Xu et al., "Serratia nematodiphila sp. nov., associated symbiotically with the entomopathogenic nematode Heterorhabditidoides chongmingensis (Rhabditida: Rhabditidae)," International Journal of Systematic and Evolutionary Microbiology, vol. 59, no. 7, p. 2646, 2009.

[26] A. Nemec, M. Musilek, O. Sedo et al., "Acinetobacter bereziniae sp. nov. and Acinetobacter guillouiae sp. nov., to accommodate Acinetobacter genomic species 10 and 11, respectively," International Journal of Systematic and Evolutionary Microbiology, vol. 60, no. 4, Part 4, pp. 896-903, 2010.

[27] H. Fang, A. K. Ohlsson, M. Ullberg, and V. Ozenci, "Evaluation of species-specific PCR, Bruker MS, VITEK MS and the VITEK 2 system for the identification of clinical Enterococcus isolates," European Journal of Clinical Microbiology \& Infectious Diseases, vol. 31, no. 11, pp. 3073-3077, 2012.

[28] E. de Jong, A. S. de Jong, N. S.-v. den Berg, and R. J. Rentenaar, "Differentiation of Raoultella ornithinolyticalplanticola and Klebsiella oxytoca clinical isolates by matrix-assisted laser desorption/ionization-time of flight mass spectrometry," Diagnostic Microbiology and Infectious Disease, vol. 75, no. 4, pp. $431-433,2013$.

[29] A. Jeffery-Smith, S. K. Taori, S. Schelenz et al., "Candida auris: a review of the literature," Clinical Microbiology Reviews, vol. 31, no. 1, 2018.

[30] R. Hajjar, G. Ambaraghassi, H. Sebajang, F. Schwenter, and S. H. Su, "Raoultella ornithinolytica: emergence and Resistance," Infection and Drug Resistance, vol. 13, pp. 1091-1104, 2020.

[31] CLSI, Performance Standards for Antimicrobial Susceptibility Testing, P. A. Wayne, Ed., Clinical and Laboratory Standards Institute, 30th edition, 2020.

[32] E. D. Perakslis, "Cybersecurity in health care," The New England Journal of Medicine, vol. 371, no. 5, pp. 395-397, 2014.

[33] J. Griffith, C. H. Bozio, A. J. Poel et al., "Imported toxinproducing cutaneous diphtheria - Minnesota, Washington, and New Mexico, 2015-2018," MMWR. Morbidity and Mortality Weekly Report, vol. 68, no. 12, pp. 281-284, 2019. 